Kalpa Publications in Computing
Volume 6, 2018, Pages 54-60
WDAM-2017. Workshop on Data Analysis in Medicine

\title{
Information Support Features of the Medical Research Process Conduction in a Huge Net Laboratory
}

\author{
I.N. Karpukhin, V.B. Yashkin \\ Laboratory "Gemotest" \\ Oktyabrskiy pr-t, 1, BC "Lermontovskiy", Lyubertsyi, 140000, Russia \\ Ilya.Karpukhin@gemotest.ru, Vladimir.Yashkin@gemotest.ru
}

\begin{abstract}
Medical laboratory "Gemotest" is a modern high-tech research center, which daily fulfills tens of thousands of medical analyses for patients all over in Russia. Regular tech base modernization and introduction of fundamentally new research methods and equipment allow "Gemotest" to fulfill widest range of analyses from clinical blood test to detection of genetic pathologies. One of the most important aspect during research conduction is instant detection of abnormal results and their verification and validation.
\end{abstract}

Keywords: Laboratory researches, Laboratory information system (LIS), expert rules, expert systems.

\section{Introduction}

Medical laboratory "Gemotest" is a modern high-tech research center, which daily fulfills tens of thousands of medical analyses for patients all over in Russia.

Today "Laboratory Gemotest" company includes about 430 laboratory offices (LO), in those there are implemented biomaterial sampling and reception. Afterwards those samples are transported to the central manufacturing laboratory complex which occupy $3500 \mathrm{M}^{2}$ area.

On the average (in according with last year data) in the laboratory there are fulfilled 3200000 analyses per month and 2500000 patients visit laboratory offices during a year.

Regular tech base modernization and introduction of fundamentally new research methods and equipment allow "Gemotest" to fulfill widest range of analyses such as:

1. General clinical analyses

2. Clinical chemistry and immunology tests

3. Microbiology tests

4. Molecular biological tests

5. Morphological test 
Each of these analyses requires special information system support.

\section{Information support of laboratory processes}

\subsection{Corporate information system landscape}

There are four main subsystems of corporate information system which one can describe from the point of view of basic laboratory manufacturing processes support

1. Gemosystem. It is a web portal which provides corporate sales and retail registration and viewing analyses result. Moreover, it provides different adjacent processes. This system is own developed by IT department. All company clients have access to this system. Patients have access to analyses results, corporate clients additionally to order registration.

2. 1C: Plant Management. It is bookkeeping and enterprise resource management system of the company

3. ISoft Laboratory. It is LIS, which allows to fulfill all client orders, send tasks to the analyzers, receive results from analyzers, keep and print results in different forms.

4. Deductor [1]. It is BI system, which is filled with data from previously mentioned systems.

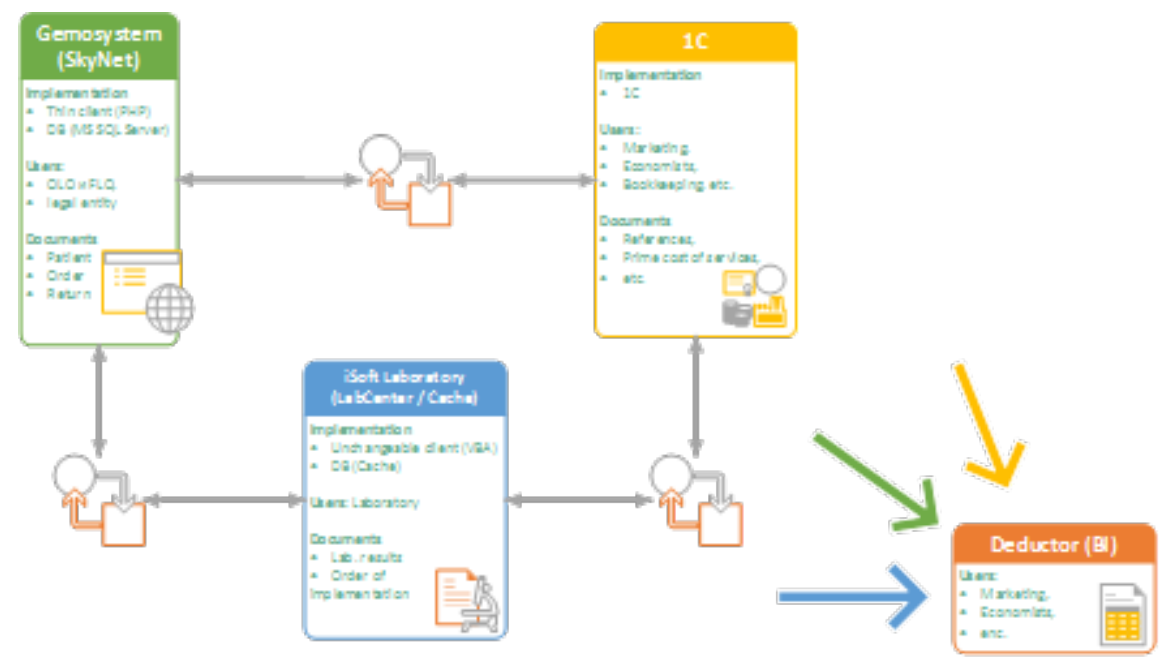

Fig. 1. Diagram of corporate system landscape

\subsection{Laboratory research production features}

Different kinds of laboratory researches are able to be produced by completely various ways. On the one hand, for most clinical chemistry tests it is sufficient put biomaterial sample into the analyzer and send clear for the device task code. In the case of successfully made previous steps the analyzer fulfils all necessary actions and returns test results as result tables. Thus, such tests are done fully automatically and their results have determined and known in advance form. On the other hand, most microbiology 
tests require qualified specialist (microbiologist) care and decision making during the progress of analyses. View of the test result depends on the way which was chosen by microbiologist. As we see, process of fulfilling tests depend on kind of test. So, there were developed a number of different units for clinical chemistry, microbiology, histology and cytology

Definitely, in both cases there are abilities of process automatization. But all those automatization attempts are restricted with automat competence. It cannot make intellectual decisions yet, so our task is at least to minimize amount of cases of human intervention needs into process. One of possible ways to decide this problem is development of intellectual rules of results validation.

\subsection{Features of laboratory equipment connection to LIS}

Gemotest is a large laboratory, so it has a lot of analyzers from tens of various vendors on its production area. Use of international standards, such as HL7 [2], allows simplify connection process of the new equipment to LIS. However, sometimes it is more convenient to use software for the group device management (middleware). In such cases there are developed connections of middleware to LIS (Fig. 2).

\section{Laboratory tests validation}

One of the highest priority tasks of the company is permanent grow of the provision of services quality. Because of this reason it is so important to not allow doubtful results for the patients.

As in any other production, laboratory researches production can suffer different failures, however, in medical cases such failures may conduct to highly unwanted consequences. Failure reasons can be quite different: violation of the rules for sampling and transporting biomaterial, analyzer calibration failure, violation of the rules of preparation for medical research by patient, etc. Not depending on these reasons, it is highly important for the laboratory to determine the failure in time in each order and to not let doubtful results provision.

To avoid doubtful results provision there are introduced validation abilities into the system. It means that in the case of doubtful result determination there is a human intervention is required. There are base rules of setting results into the validation state such as when analyzer sends flags of unreliable result or result value exceeds the normal range. In the first case there is a technical validation example, and second case describes medical validation. However, it has been found out that those validation rules are not sufficient for determination of all kinds of doubtful results. So the additional validation mechanism for clinical chemistry has been developed in the company. 


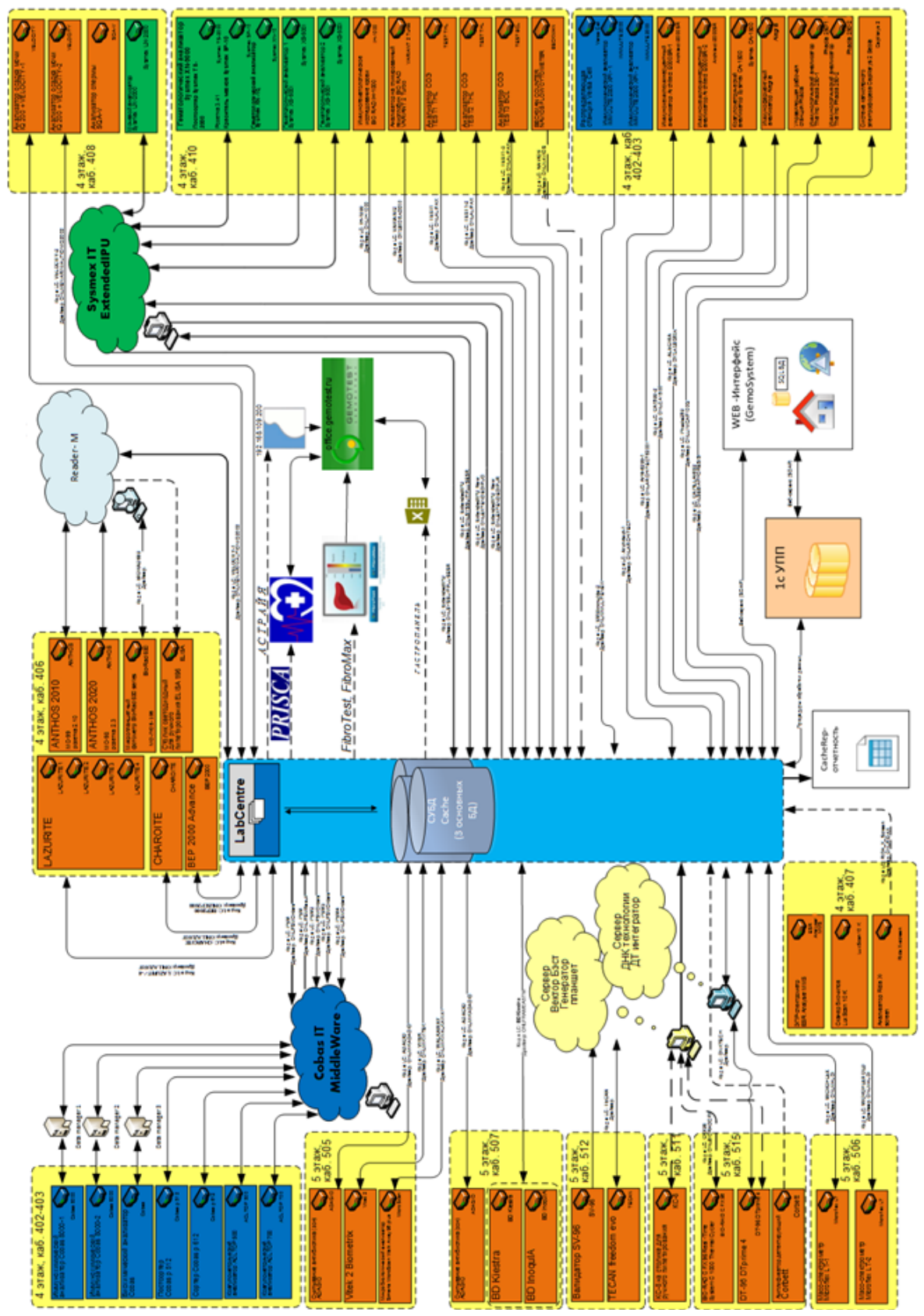

Fig. 2. Top level scheme of connection some laboratory devices to LIS

\subsection{Additional validation levels}

Learning cases of technical and medical validation it became clear that single result mark which has to be considered correct according to the rules of validation actually is abnormal in correlation with other results marks in this or previous patient's orders. For example, normal (in the limits of normal values) 
values of alanine aminotransferase and aspartate aminotransferase are abnormal at simultaneous positive value of hepatitis $\mathrm{C}$ test. Such values have to be evaluated by laboratory doctor, who in his turn may appoint additional researches or ask a patient to give another biomaterial sample.

To provide use of such rules the description mechanism has been offered. It uses predicates described in the form of reverse Polish entry. Validation rules are kept in the elements of the test catalog. For example of the rule which sounds like: "Output to the additional level of validation in the case of obtaining urea values above the reference values, and creatinine values within the reference values" there is such formula:

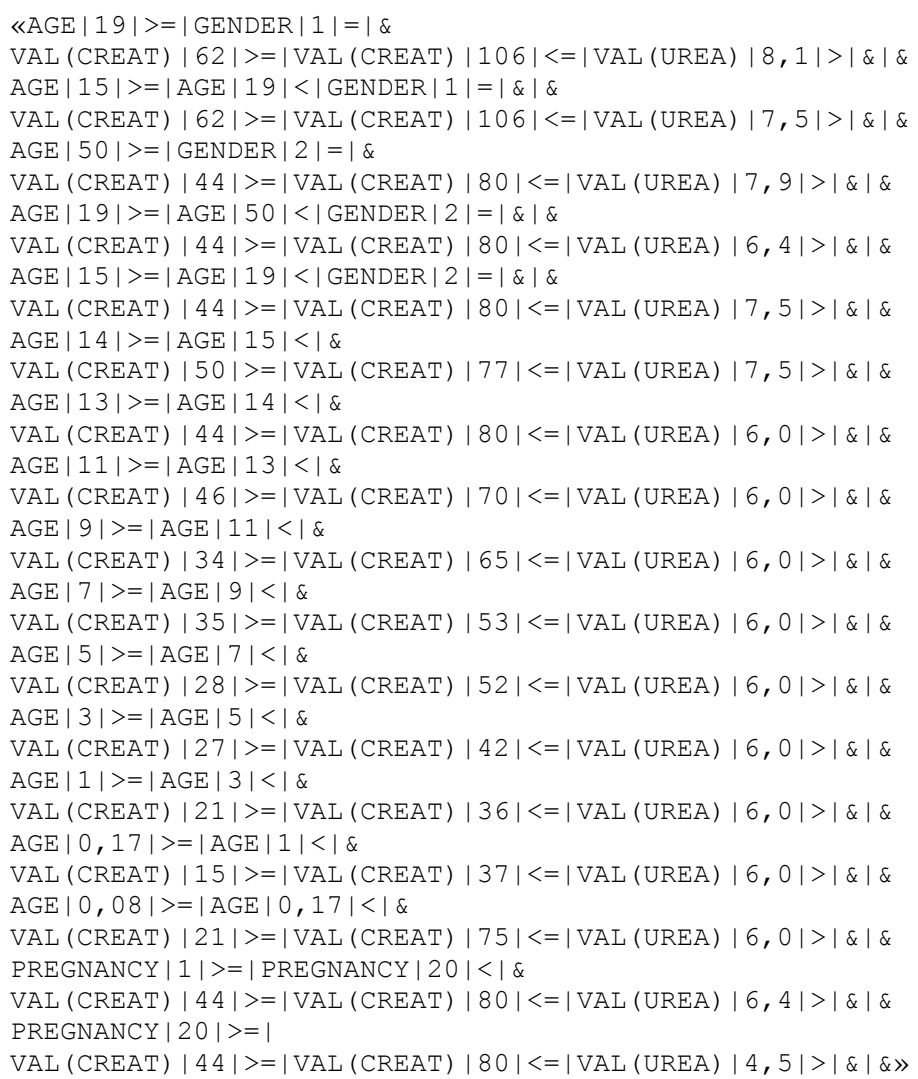

Finally, between two points of getting results from laboratory device and sending it to client there can be stop point caused by different reasons. Depending on amount of allocated levels we can set some number of stop points. For each point according to validation level appropriate laboratory employee is responsible (Fig. 3). 


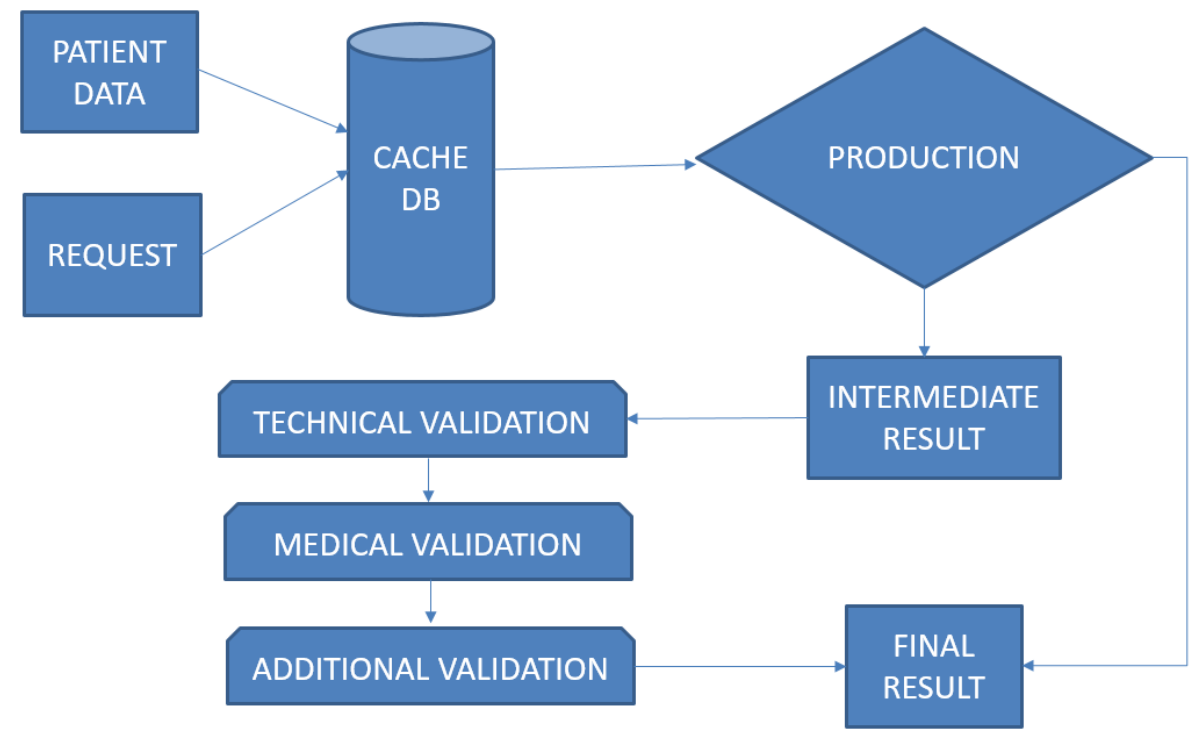

Fig. 3. Validation levels of laboratory tests

\subsection{Additional level validation for microbiology researches}

Microbiology researches have their own particularity of providing reliability of received results. Common sense is the same as in clinical chemistry. There must be no contradictory results. Quality of the validation is determined by the probability of contradictory results in final conclusion. In contrast to clinical chemistry there are French microbiology community (SFM standard), the European Committee on the Determination of Antibiotic Sensitivity (EUCAST standard [3]) and others, who develop interpretation and transformation antibioticogram rules. In laboratory Gemotest there are installed equipment and software of Becton, Dickinson and Company, which are able to download new rules, approved by international standards of processing antibiotic results [4].

\section{$4 \quad$ Laboratory tests and services classification}

Currently developed validation rules influence on the production of laboratory researches of clinical chemistry by using own catalog of services, which consist of over 3000 different items. Despite of partner's demands to use federal and international catalogs of laboratory services in existing integrations, we met some difficulties in joining own catalog with other catalogs. We developed the instrument which permits to bind each item of our catalog with items of FSLI [5] and LATEUS [6]. Both of them actually are the adaptation and extension of LOINC [7] - international universal standard of medical and laboratory researches. However, during the binding we met such problems as:

1. Not all of the partners are ready to work with those catalogs;

2. There are test in Gemotest catalog which have no evident correlation;

3. There are cases when some different in production Gemotest catalog items correlate with single LOINC item (for example, the case when a test are made from different biomaterials); 
4. As presented catalogs contain new items (not presented in LOINC) such cases appear, when the same items of FSLI and LATEUS correlate with different tests.

We consider first of all, that these problems appear because of absence of unified classification standard of laboratory tests and services in Russia. While this question is not resolved, mentioned problems are stay actual.

\section{$5 \quad$ Future work}

Intention of permanent quality grow of accorded services sets new tasks for IT development within company and more often these tasks are bound with intellectual analysis of accumulated data, Big Data algorithms and machine learning. The demand appears of detection of new validation rules, which allow finding hidden now anomalies. Also appears question of automated evaluation of validating doctors work quality.

\section{References}

1. BaseGroup Labs (https://basegroup.ru/).

2. HL7 System \& Services (http://www.hl7.com.au/).

3. European Committee on Antimicrobial Susceptibility Testing (http://www.eucast.org/).

4. BD Medical Technology (http://www.bd.com/)

5. Federal Handbook of laboratory researches. Handbook of laboratory tests (https://nsi.rosminzdrav.ru/\#!/refbook/1.2.643.5.1.13.13.11.1080).

6. Medical Information and analytical center. Data exchange of laboratory researches in S.Peterburg (http://spbmiac.ru/odli/).

7. The universal standard for identifying health measurements, observations, and documents (https://loinc.org/). 